\title{
DOCUMENTOS PARA LA HISTORIA DE MAGALLANES
}

\author{
SOCIEDAD AURIIFERA "NAVARINO", BAHÍA WINDHOND (1905) \\ Introducción y notas \\ Samuel García Oteiza ${ }^{a}$
}

La extracción aurífera mecanizada comenzó a desarrollarse en Tierra del Fuego y en la región del Beagle y Cabo de Hornos durante la primera década de 1900. Con la implantación de esta nueva tecnología (dragas, pistones hidráulicos, palas a vapor, etc.) se esperó extraer cantidades considerables del preciado metal, superando con creces la productividad que la explotación de manera artesanal había permitido desde fines del siglo XIX hasta entonces. El panorama era alentador y expertos en la materia declaraban anticipadamente el éxito y las ventajas de la aplicación de este nuevo método de extracción. Consecuencia directa de esta nueva posibilidad que ofrecía la tecnología fue la constitución entre los años 1900-1907 de aproximadamente 34 sociedades auriferas, los estatutos de varias de ellas fueron aprobados mediante decretos supremos pero nunca llegaron a operar en terreno (Martinic, 2003, p. 238) ${ }^{1}$.

En lo que respecta a las islas subantárticas se conocen al menos dos compañías con propiedades auriferas en la orilla sur del canal Beagle; Compañía Aurífera de Lennox (1905) y Compañia Aurífera Slava (1907). De las entidades mencionadas, al menos de la primera existen antecedentes que operó (Op. cit., p. 242; Martinic, 2005, pp. 95-96), respecto a la segunda podemos indicar que los lavaderos de oro que pretendía explotar, según un mapa construido por la compañía, estaban en la bahía Ortega, ubicada en la vecindad de la bahía Windhond, litoral sur de la isla Navarino ${ }^{2}$. Es importante señalar como antecedente la existencia de la compañía The Fortunato Beban Exploration Company, cuya organización se publicitó en 1905 y sobre la que no hay más referencia que su denominación y la vinculación que sugiere la inclusión del nombre de Fortunato Beban de quien se sabe fehacientemente que en la época ocupaba al igual que Oreste Grandi, una sección de los campos fiscales situados en la bahía Windhond (García, 2016, pp. 17, 377; Martinic, 2005, pp. 117-122). Con respecto a la ubicación de sus propiedades auríferas, en el caso de haber existido, podríamos conjeturar que estaban situadas en algún punto de la bahía Windhond y/o alrededores, lugares que presentaban buenas expectativas para fines extractivos auríferos.

El texto que transcribimos en esta entrega

a Arquitecto. Investigador del Centro de Estudios del Hombre Austral, Instituto de la Patagonia, Universidad de Magallanes. samuelgarciaoteiza@gmail.com

1 Ver también: www.patlibr.org/oro

2 Esta compañía fue fundada en 1907 en Santiago y su principal accionista fue Pascual Baburizza (Martinic, 2005, p. 95). El mapa construido por la Compañía Aurífera Slava (Isla Navarino/ Dibujó: Ramón de Lartundo F./
Escala 1:125.000/Junio 1907) para indicar la ubicación de sus lavaderos puede ser consultado en su formato digital en la mapoteca digital de la Biblioteca Nacional de Chile (www. bncatalogo.cl, código de búsqueda: 001106511). 
corresponde a un prospecto que propone la formación de la Sociedad Aurífera Navarino, Bahía Windhond, publicado aparentemente en $1905^{3}$ e impreso en Santiago por la imprenta El Globo ${ }^{4}$.

Este documento se inserta en el auge de la fase mecanizada de la extracción aurífera, momento en el cual los trabajos con los nuevos sistemas de dragas estaban estrenándose en la región norte de Tierra del Fuego.

Como es común en este tipo de escritos, el objetivo era publicitar a la empresa favorablemente, con argumentos convincentes para captar accionistas entendidos y no en la materia. Una de las principales ventajas que distinguía a la compañía de otras, y es resaltada en el prospecto, era lo referido a la logística de dos factores importantes: el acceso terrestre al lugar de extracción y el abastecimiento de combustible para las dragas. El hecho de estar emplazadas las propiedades auríferas en el borde litoral de la bahía Windhond significaba un ahorro importante de inversión. En primer lugar por no tener la necesidad de construir caminos y además porque el combustible para el funcionamiento de las maquinas sería obtenido de la explotación de los bosques, que supuestamente, se ubicaban en la vecindad de la bahía. De esta manera se expuso la ventaja que existía sobre otras compañias, realizándose una comparación con la Compañía Sutphen de Lavaderos de Oro de Tierra del Fuego, Compañía de Dragaje de Rio de Oro y Compañía de Dragaje Río Verde (ambas emplazadas en el norte de Tierra del Fuego) que, en el caso de la primera, tuvo que invertir una importante cantidad de recursos económicos en la apertura de caminos para trasladar los elementos que componían la draga entre el puerto de desembarque y el lugar de emplazamiento ubicado al interior del territorio. Así también, se advierte la cantidad de dinero que requeririan invertir las otras dos compañías que próximamente deberían realizar la misma operación. Otro punto al que se hace mención en el prospecto, corresponde a una positiva descripción técnica del funcionamiento

3 En el documento original que hemos tenido a la vista, no estaba indicado el año de publicación de forma impresa, pero en su portada y con lápiz tinta estaba escrito el año 1905.

4 Dimensiones $22,3 \times 15,5 \mathrm{~cm}$. Al final del texto incluye un y rendimiento de esta nueva propuesta de explotación avalada y argumentada de forma cuantitativa.

Un antecedente que no pasa inadvertido en el texto es la cantidad de pertenencias auríferas que disponía la compañía. Según el documento, la Sociedad Aurifera Navarino, Bahía Windhond poseía 308 pertenencias de arenas auríferas situadas en las playas de la bahía Windhond adquiridas por Rómulo Correa, Froilan González y Fabián Martínez. Como forma de resaltar tan llamativo y tentativo número, éste fue indicado, las dos veces que fue mencionado, entre comillas y destacado con negrita. La cuestión de la cantidad de pertenencias auríferas era una estrategia publicitaria recurrente por parte de los gestores de las sociedades auríferas. El Gobernador del Territorio de Magallanes de la época Alberto Fuentes se refirió a este tema, exponiendo que la especulación y la cantidad desmedida de pertenencias auríferas, muchas de ellas alejadas de la realidad, eran algunos de los principales motivos del fracaso de éste nuevo método de explotación (Martinic, 2003, pp. 237-239).

Si bien el documento que desclasificamos en este entrega fue publicado, hasta ahora no existía ningún tipo de mención sobre él en estudios sobre la materia (Navarro, 1908; Martinic, 2003, 2005), lo cual nos lleva a pensar que más que un prospecto en forma referido al negocio aurífero y a la correspondiente organización de una sociedad anónima para emprender su explotación, habría sido un volante informativo preliminar ${ }^{5}$ destinado al ambiente de los posibles inversionistas para sondear su interés y, supuesta una escasa acogida, que la pretendida Sociedad Aurífera Navarino, Bahía Windhond nunca llegó a constituirse, no definitivamente en el curso de 1905 y el de los siguientes años, por ausencia de toda mención a la misma en la crónicas de la época, como se aprecia en los exhaustivos estudios citados más arriba.

Aunque breve, el prospecto de la Sociedad Aurífera Navarino, Bahía Windhond nos permite

mapa (dimensiones $22,3 \times 30,5 \mathrm{~cm}$ en blanco y negro), ver Fig. 1.

5 Igualmente desconocemos los alcances y lugares de difusión de este documento. 
conocer las pretensiones de los capitalistas de la época, entregando una interesante información sobre el desarrollo, competencia y acontecer aurífero desde el discurso publicitario de las empresas explotadoras. Si bien no disponemos de antecedentes sobre si la sociedad en cuestión logró constituirse y luego operar, así como también si tuvo alguna relación con la mencionada The Fortunato Beban Exploration Company, con la cual comparte el año de publicación (1905) y posiblemente el lugar de extracción (bahía Windhond), creemos que es de interés incorporarla en las próximas investigaciones referidas a la explotación aurífera en las islas subantárticas, así como también poder conocer y analizar los prospectos de otras sociedades del rubro que se formaron paralelamente $y$ con intereses en la región. De esta forma se podría tener una lectura amplia y crítica del proceso mecanizado de extracción, que por casi una década (1903-1910) sustentó un sueño cuyo desenlace fue caminos $^{6}$ y maquinarias abandonadas.

En cuanto a la transcripción del documento, cabe señalar que hemos conservado la gramática original y reproducido el mapa que incluye (Fig. 1).

\section{—SOCIEDAD AURIFERA "NAVARINO"- "BAHÍA WINDHOND" 1905}

\author{
Directorio \\ Presidente: \\ Don Eulogio Guzmán \\ Directores:
}

Don Aníbal Rodríguez $H$.

6 La apertura de caminos fue una necesidad vital para introducir los grandes equipos industriales desde los lugares de desembarco hasta los puntos de extracción. Así ocurrió con el camino abierto entre el puerto de Porvenir y el cordón Baquedano a comienzos de 1904; Personas llegadas de Tierra del Fuego nos aseguran que ya están próximos a terminarse los trabajos del camino que debe unir el pueblo de Porvenir con los yacimientos auríferos que en algún tiempo más empezarán a explotar la The Sutphen Gold Fields Co., en el punto en que se une el chorrillo Baquedano al Río del Oro. El camino tiene 21 millas de extensión $y$ es de primer orden y apto para toda clase de vehículos. Con la conclusión de esta importante obra de vialidad pública, vendrá a coincidir la llegada de una draga, que ya viene en camino de Nueva York

\section{Don Juan Estéban Errázuriz \\ Don Rómulo Correa C. Don Froilán González}

\author{
Secretaría: Agustinas, 940 \\ Casa M. Vidaurrázaga \\ Capital: $£ 100.000$
}

Dividido en 100,000 acciones de á una libra

\section{SOCIEDAD AURIFERA "NAVARINO" "BAHÍA WINDHOND"}

Hace aproximadamente veinte años que se está trabajando en la explotación de terrenos auríferos en Navarino, Lennox y Sloggett (Territorio Argentino) por procedimientos primitivos, sin elementos ni maquinarias y á pesar de efectuar los trabajos en esta forma, han visto coronados sus esfuerzos los que á ellos se han dedicado, con resultados positivos.

La sociedad que hoy propone para explotar con dragas, "último invento", tiene por base las propiedades auriferas que se adquieren de Don Rómulo Correa, Don Froilán González y Don Fabián Martínez, en todo “308» pertenencias manifestadas y registradas convenientemente en Punta Arenas y ubicadas en la Isla Navarino, playa de Bahía Windhond, según el plano demostrativo que se acompaña [Fig. 1].

Prevenimos á los accionistas que la extensión y calidad de los terrenos con que se funda la Sociedad, los cuales, puede decirse, han sido los primeros descubiertos y explotados desde que se inició en Magallanes la explotación aurifera, satisfacen cumplidamente las

por uno de los vapores Merchant's Line, destinada a la empresa ya nombrada [...] (El Comercio, 23 de enero 1904). A consecuencia de la decadencia y término de esta práctica extractiva del material preciado, los caminos quedaron sujeto al abandono y fue un tema que preocupó a la autoridades regionales; Subdelegación de Tierra del Fuego- Vialidad: Habría conveniencia en que se consulte alguna suma de dinero para la conservación de los caminos rurales que, como se sabe, fueron arreglados por cuenta de las diferentes sociedades mineras $i$ que, debido a la paralización de las operaciones de éstas, han quedado en completo abandono. "Memoria del Gobernador de Magallanes". En: Memoria del Ministerio del Interior correspondiente al año 1910,136 , p. 8. Archivo Nacional Histórico (DIBAM). 
esperanzas de un magnífico negocio, como á grandes rasgos vamos á demostrarlo.

En la forma ó sistema de trabajos primitivos de canaleta y chaya, un obrero extraía en seis horas de trabajo manual y contínuo, en los terrenos de que hablamos, como mínimum, de tres á cuatro gramos de oro.

Con el nuevo sistema de dragas, cuyos trabajos en breve se van á iniciar en Río de Oro y Río Verde, maquinarias que empezarán a funcionar á lo más tardar, á mediados de Enero y cuyo resultado no podrá ser sino muy favorable como lo ha sido en diversos países que las tienen en explotación, la industria aurífera tomará un inmenso incremento.

Hay dragas que remueven 60 toneladas de arena por hora y otras que remueven 100, pudiendo llegar ambas hasta una profundidad de veinte metros y cuyo lecho se lo cavan ellas mismas. Es natural y lógico suponer que no habiendo hasta la fecha trabajado sino en la superficie y con los resultados espléndidos ya indicados, conforme aumente la profundidad del trabajo asi el rendimiento será gradualmente mucho mayor, pues es cosa naturalmente conocida que el oro por su mismo peso sobre las arenas se va profundizando hasta encontrar la circa.

Creemos que el tipo de draga que debe adoptarse para los trabajos de esta Sociedad es de las dragas de último sistema que remueven 60 toneladas por hora con un personal de 11 hombres, de administrador á cocinero.

Aunque estas dragas pueden trabajar

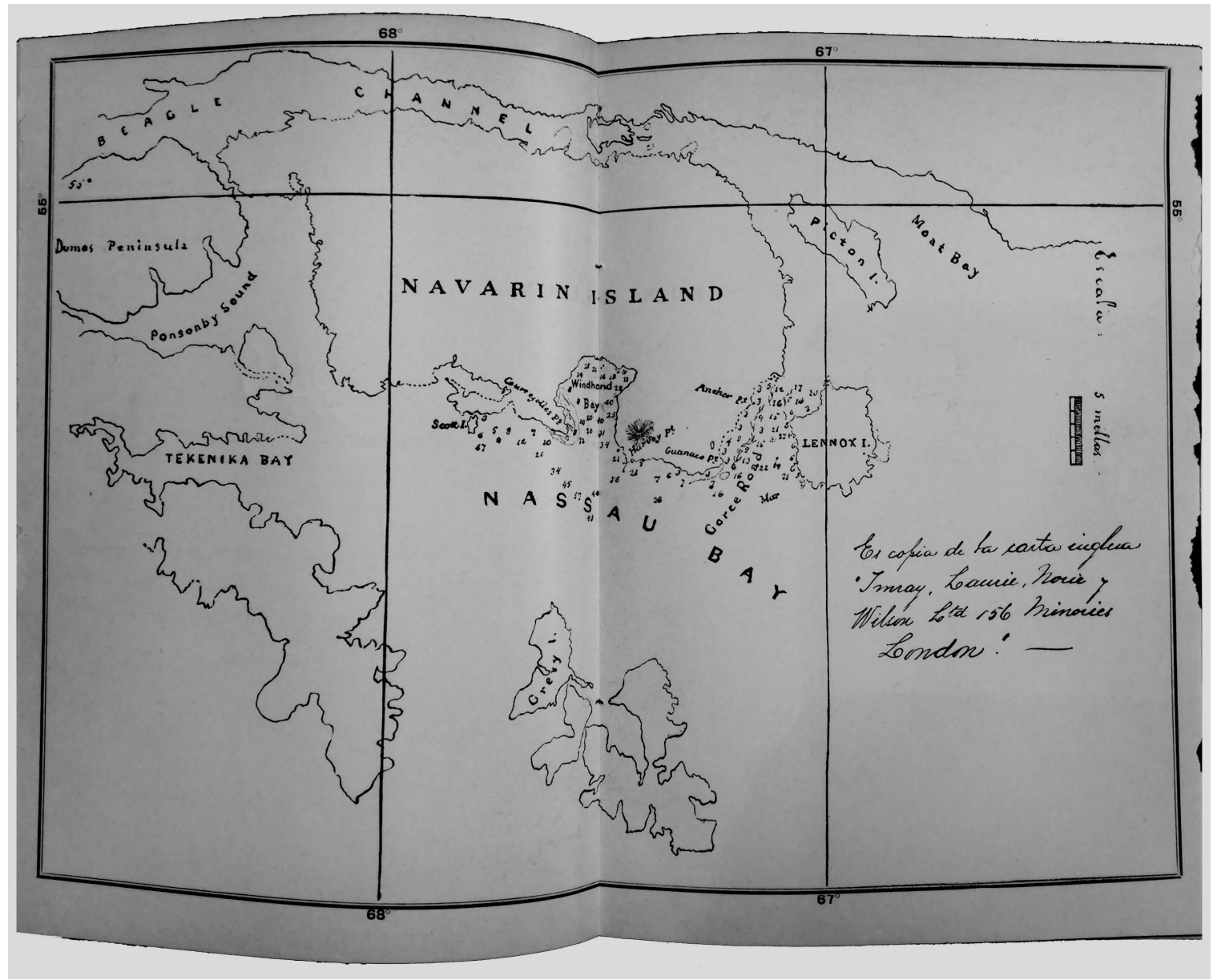

Fig. 1. Mapa incluido en el prospecto. En su costado derecho se indica: Es copia de la carta inglesa "Imray, Laurie, Norie y Wilson Ltd 156 Minories London" (Formato 30,5 x 22,3 cm). 
día y noche, juzgamos lo más práctico hacerlas trabajar diez horas diarias y sobre esta base entablamos el cálculo. Calculando muy por lo bajo á razón de dos gramos de oro por cada tonelada de arena de la superficie, resulta que en diez horas de trabajo, una draga lavará 600 toneladas de arenas auríferas que arrojan un producto de 1.200 gramos, que valorizándolos tan sólo á un peso cincuenta centavos gramo, daría un resultado total de 1.800 pesos por día. Si se optase por trabajar día y noche se aumentaría el personal en ocho hombres más, que sería la cuadrilla nocturna y el rendimiento, como es lógico, sería proporcionalmente mayor.

El hecho de estar ubicadas las « $\mathbf{3 0 8} »$, todas continuas, en Isla Navarino, Bahía Windhond, reporta á la Sociedad gran economía en tiempo y capitales.

La Bahía Windhond es un punto de abrigo por esas regiones y facilita el desembarco de esta gran maquinaria en la playa misma donde ha de empezar sus trabajos, evitando asi el tener que hacer grandes caminos para su conducción como ha sucedido á Río Verde y Río de Oro, cuyas sociedades invertirán no menos de 60.000 pesos en hacer caminos y transportar su maquinaría, caso que ha ocurrido igualmente a la Compañia Sutphen (Argentina) que ha gastado más de 100.000 pesos en transportar su maquinaria a Río Baquedano.

Otra gran economía para la explotación significa tener el combustible necesario para las dragas a menos de 800 metros de la playa, pues las sociedades Río Verde, Río Oro, Sutphen y cualquiera otra que se formara en estos territorios tendría que usar carbón, por no haber en estos parajes leña, cuyo costo es de mucha importancia, lo que demuestra, como indicamos más arriba, el menor costo para la explotación que conseguirá la Sociedad Navarino "Bahía Windhond" sobre las demás análogas.

El capital social es de $£ 100.000$ dividido en 100.000 acciones de una libra cada una, de las cuales 50.000 acciones que se estiman totalmente pagadas se aplicarán al pago de los terrenos auríferos que se adquieren de los Sres. D. Rómulo Correa, D. Froilán González y D. Fabián Martínez.

Las $£ 50.000$ de obligación se pagarán como sigue:

$1^{\circ}$ cuota al suscribirse la escritura pública, de $1 / 2$ libra. $1 / 2$ libra.

$2^{\circ}$ cuota cuando lo estime el Directorio,

\section{BIBLIOGRAFÍA}

García, S. (Comp.) (2016). Isla Navarino: documentos inéditos 1896-1956. Punta Arenas: Entrepáginas.

Martinic, M. (2003). La minería aurífera en la Región Austral americana (1869-1950). Historia, 36, 219-254.

Martinic, M. (2005 [1973]). Crónica de las tierras al sur del canal Beagle. Punta Arenas: Lakutaia.

Navarro, L. (1908). Censo Jeneral del Territorio de Magallanes. Tomo II. Punta Arenas: s/e. 
